\title{
Some Alternative Classes of Shrinkage Estimators for a Scale Parameter of the Exponential Distribution
}

\author{
Housila P. Singh ${ }^{1}$. Sarjinder Singh ${ }^{2}$. Jong-Min Kim ${ }^{3}$ \\ ${ }^{1}$ School of Studies in Statistics, Vikram University \\ ${ }^{2}$ Department of Mathematics, Texas A\&M University-Kingsville \\ ${ }^{3}$ Statistics Discipline, University of Minnesota at Morris
}

(Received April 22, 2011; Revised February 16, 2012; Accepted March 5, 2012)

\begin{abstract}
This paper proposes some alternative classes of shrinkage estimators and analyzes their properties. In particular, some new shrinkage estimators are identified and compared with Pandey (1983), Pandey and Srivastava (1985) and Jani (1991) estimators. Numerical illustrations are also provided.

Keywords: Shrinkage estimators, prior information, scale parameter, bias, mean squared error.
\end{abstract}

\section{Introduction}

The exponential model is widely used in the field of life testing and reliability. Let $x_{1}, x_{2}, \ldots, x_{n}$ be a random sample from exponential distribution with the probability density function

$$
f(x, \theta)=\frac{1}{\theta} \exp \left\{-\left(\frac{x}{\theta}\right)\right\}, \quad \theta>0, x \geq 0 .
$$

The maximum likelihood estimate for the scale parameter $\theta$ is the sample mean $\bar{x}=\left(\sum_{i=1}^{n} x_{i} / n\right)$ which is unbiased and the minimum variance unbiased linear estimate. The minimum mean squared error(MMSE) estimator of $\theta$ in the class of linear estimators of the type $\sum_{i=1}^{n} c_{i} x_{i}$ is

$$
T_{1}=\frac{n}{n+1} \bar{x}
$$

with mean squared error(MSE)

$$
\operatorname{MSE}\left(T_{1}\right)=\frac{\theta^{2}}{n+1} .
$$

In many practical situations the experimenter has some prior estimates regarding the value of the parameter, either due to experience or acquaintance with the behavior of the system. Let $\theta_{0}$ be

${ }^{3}$ Corresponding author: Statistics Discipline, University of Minnesota at Morris, Morris, MN 56267, USA.

E-mail: jongmink@morris.umn.edu 
the guessed value or prior estimate of $\theta$. Thompson (1968) gave the idea of shrinking the minimum variance unbiased estimator(MVUE) towards the prior estimate $\theta_{0}$, in order to divide a better estimate and suggested a shrinkage estimator

$$
T_{2}=K \bar{x}+(1-K) \theta_{0}
$$

where $K$ is a known constant, $0<K<1$, and is specified by the experimenter in advance according to his belief in prior estimate $\theta_{0}$. A value of $K$ near to one implies strong belief in sample values. If $K$ is not known, Pandey (1983) suggested two shrunken estimators for $\theta$ as

$$
T_{j}=\frac{\left(\bar{x}-\theta_{0}\right)^{3}}{\left\{\left(\bar{x}-\theta_{0}\right)^{2}+\frac{\bar{x}^{2}}{n+j-3}\right\}}+\theta_{0} ; \quad(j=3,4) .
$$

Pandey (1983) has shown that $T_{j},(j=3,4)$ have smaller MSE than MMSE estimator $T_{1}$ if $|r| \leq 0.3$, where $r=\left\{\left(\theta_{0} / \theta\right)-1\right\}$.

Pandey and Srivastava (1985) proposed an estimator for $\theta$ as

$$
T_{5}=\theta_{0}+\left(\frac{n-2}{n}\right)\left(\bar{x}-\theta_{0}\right)
$$

and claimed that for large samples $T_{5}$ has smaller MSE than $T_{1}$ if $|r| \leq \sqrt{3} / 2$.

Pandey and Srivastava (1985) suggested two more estimators for $\theta$ as

$$
T_{6}=\theta_{0}+\left(\frac{n-1}{n}\right)\left(\bar{x}-\theta_{0}\right)
$$

and

$$
T_{7}=\theta_{0}+\left(\frac{n^{2}+n-12}{n^{2}}\right)\left(\bar{x}-\theta_{0}\right)
$$

Jani (1991) suggested a class of estimators for $\theta$ as

$$
T_{p}=\theta_{0}\left\{1+W\left(\frac{\theta_{0}}{\bar{x}}\right)^{p}\right\}
$$

where $W$ is a constant such that MSE of $T_{p}$ is minimum and $p$ is a 'non-zero' real number. The MSE of $T_{p}$ is given by

$$
\operatorname{MSE}\left(T_{p}\right)=\theta^{2}\left\{r^{2}+W^{2}(1+r)^{2(p+1)} \frac{n^{2 p} \sqrt{n-2 p}}{\sqrt{n}}+2 W r(1+r)^{(p+1)}-\frac{n^{p} \sqrt{n-p}}{\sqrt{n}}\right\} .
$$

Minimization of (1.10) with respect to $W$ gives

$$
W=\left(\frac{\theta-\theta_{0}}{\theta}\right)\left(\frac{\theta}{\theta_{0}}\right)^{(p+1)} \frac{\sqrt{n-p}}{n^{p} \sqrt{n-2 p}} .
$$

Since (1.11) contains unknown parameter $\theta$, thus we cannot know $W$. Replacing $\theta$ by its MLE/MVUE $\bar{x}$, Jani (1991) obtained the estimate of $W$ as

$$
\widehat{W}_{1}=\left(\frac{\bar{x}-\theta_{0}}{\bar{x}}\right)\left(\frac{\bar{x}}{\theta_{0}}\right)^{(p+1)} \frac{\sqrt{n-p}}{n^{p} \sqrt{n-2 p}} .
$$


Substituting (1.12) in (1.9), Jani (1991) obtained a class of estimators for $\theta$ as

$$
T_{p_{1}}=\theta_{0}+W_{p(1)}\left(\bar{x}-\theta_{0}\right)
$$

with

$$
W_{p(1)}=\frac{\sqrt{n-p}}{n^{p} \sqrt{n-2 p}} .
$$

For $p=1$, (1.13) reduces to Pandey and Srivastava (1985) estimator $T_{5}$ while $p=-1, T_{p_{1}}$ reduces to the estimator

$$
T_{8}=\theta_{0}+\left(\frac{n}{n+1}\right)\left(\bar{x}-\theta_{0}\right)
$$

which is due to Jani (1991).

Many more shrinkage estimators can be obtained from (1.13) for different values of $p$. However, it should be mentioned here that the class of shrinkage estimators $T_{p_{1}}$ obtained by Jani (1991) is not unique. In this paper we have provided some new alternative classes of shrinkage estimators for $\theta$, just by giving different estimators of $W$ in (1.11).

\section{Alternative Classes of Shrinkage Estimators}

Case 1: If in (1.11), we replace $\theta$ by $\bar{x}$ and $\theta^{(p+1)}$ by its unbiased estimator

$$
\hat{\theta}_{u}^{(p+1)}=\frac{n^{(p+1)} \sqrt{n}}{\sqrt{n+p+1}} \bar{x}^{(p+1)},
$$

we get the estimated value of $W$ as

$$
\widehat{W}_{2}=\left(\frac{\bar{x}-\theta_{0}}{\theta_{0}}\right)\left(\frac{\bar{x}}{\theta_{0}}\right)^{p}\left(\frac{n}{n+p}\right) \frac{\sqrt{n} \sqrt{n-p}}{\sqrt{n+p} \sqrt{n-2 p}} .
$$

Substitution of $\widehat{W}_{2}$ in (1.9) yields the shrinkage estimator

$$
T_{p_{2}}=\theta_{0}+W_{p(2)}\left(\bar{x}-\theta_{0}\right)
$$

with

$$
W_{p(2)}=\left(\frac{n}{n+p}\right) \frac{\sqrt{n} \sqrt{n-p}}{\sqrt{n+p} \sqrt{n-2 p}} .
$$

Replacing $\bar{x}$ by MMSE estimator $(n /(n+1)) \bar{x}$ in $T_{p_{2}}$ we get the following class of shrinkage estimators for $\theta$ as

$$
T_{p_{2}}^{*}=\theta_{0}+W_{p(2)}\left\{\left(\frac{n}{n+1}\right) \bar{x}-\theta_{0}\right\}
$$

For $p=1, T_{p_{2}}^{*}$ reduces to the estimator

$$
T_{9}=\theta_{0}+\left(\frac{n-2}{n+1}\right)\left\{\left(\frac{n}{n+1}\right) \bar{x}-\theta_{0}\right\} .
$$


Case 2: If we replace $\theta$ by $\bar{x}$ and $(1 / \theta)$ by its unbiased estimator $(1 / \hat{\theta})_{u}=((n-1) / n)(1 / x)$ in (1.11) we get an estimator of $W$ as

$$
\widehat{W}_{3}=\left(\frac{\bar{x}-\theta_{0}}{\theta_{0}}\right)\left(\frac{\bar{x}}{\theta_{0}}\right)^{p+1}\left(\frac{n-1}{n}\right) \frac{\sqrt{n-p}}{n^{p} \sqrt{n-2 p}} .
$$

Substitution of $\widehat{W}_{3}$ in (1.9) gives the following class of shrinkage estimators

$$
T_{p_{3}}=\theta_{0}+W_{p(3)}\left(\bar{x}-\theta_{0}\right)
$$

where

$$
W_{p(3)}=\left(\frac{n-1}{n}\right) \frac{\sqrt{n-p}}{n^{p} \sqrt{n-2 p}} .
$$

Replacing $\bar{x}$ by MMSE estimator $(n /(n+1)) \bar{x}$ in $(2.5)$ we get another class of shrinkage estimators for $\theta$ as

$$
T_{p_{3}}^{*}=\theta_{0}+W_{p(3)}\left\{\left(\frac{n}{n+1}\right) \bar{x}-\theta_{0}\right\} .
$$

For $p=1, T_{p_{3}}^{*}$ boils down to the shrinkage estimator

$$
T_{10}=\theta_{0}+\frac{(n-1)(n-2)}{n^{2}}\left\{\left(\frac{n}{n+1}\right) \bar{x}-\theta_{0}\right\} .
$$

Case 3: Replacing $\theta$ by $\bar{x},(1 / \theta)$ by $(1 / \theta)_{u}=((n-1) / n)(1 / x)$ and $\theta^{(p+1)}$ by $\hat{\theta}_{u}^{(p+1)}=n^{p+1} \sqrt{n} /$ $\sqrt{n+p-1}$ in (1.11) we get another estimator of $W$ as

$$
\widehat{W}_{4}=\left(\frac{\bar{x}-\theta_{0}}{\theta_{0}}\right)\left(\frac{\bar{x}}{\theta_{0}}\right)^{p}\left(\frac{n-1}{n+p}\right) \frac{\sqrt{n} \sqrt{n-p}}{\sqrt{n-2 p} \sqrt{n+p}} .
$$

Putting $\widehat{W}_{4}$ in (1.9) we get a class of estimators for $\theta$ as

$$
T_{p_{4}}=\theta_{0}+W_{p(4)}\left(\bar{x}-\theta_{0}\right)
$$

where

$$
W_{p(4)}=\left(\frac{n-1}{n+p}\right) \frac{\sqrt{n} \sqrt{n-p}}{\sqrt{n-2 p} \sqrt{n+p}} .
$$

Again replacing $\bar{x}$ by MMSE estimator $(n /(n+1)) \bar{x}$ in $(2.8)$ we get a class of shrinkage estimators for $\theta$ as

$$
T_{p_{4}}^{*}=\theta_{0}+W_{p(4)}\left\{\left(\frac{n}{n+1}\right) \bar{x}-\theta_{0}\right\} .
$$

If we set $p=1 \mathrm{in}(2.9)$ we get the shrinkage estimator for $\theta$ as

$$
T_{11}=\theta_{0}+\frac{(n-1)(n-2)}{n(n+1)}\left\{\left(\frac{n}{n+1}\right) \bar{x}-\theta_{0}\right\} .
$$

Case 4: Replacing $\theta$ by its MMSE estimator $(n /(n+1)) \bar{x},(1 / \theta)$ by its MMSE estimator $((n-$ $2) / n)(1 / x)$ in (1.11) we get the estimated value of $W$ as

$$
\widehat{W}_{5}=\left(\frac{1}{\theta_{0}}\right)\left\{\left(\frac{n}{n+1}\right) \bar{x}-\theta_{0}\right\}\left(\frac{\bar{x}}{\theta_{0}}\right)^{p}\left(\frac{n-2}{n+1}\right) \frac{\sqrt{n-p}}{(n+1)^{p} \sqrt{n-2 p}} .
$$


Putting $\widehat{W}_{5}$ in $(1.9)$ we get the class of estimators for $\theta$ as

$$
T_{p_{5}}=\theta_{0}+W_{p(5)}\left\{\left(\frac{n}{n+1}\right) \bar{x}-\theta_{0}\right\},
$$

where

$$
W_{p(5)}=\left(\frac{n-2}{n+1}\right) \frac{\sqrt{n-p}}{(n+1)^{p} \sqrt{n-2 p}} .
$$

For $p=1, T_{p_{5}}$ turns out to the shrinkage estimator for $\theta$ as

$$
T_{12}=\theta_{0}+\left(\frac{n-2}{n+1}\right)^{2}\left\{\left(\frac{n}{n+1}\right) \bar{x}-\theta_{0}\right\} .
$$

Thus we see that a large number of classes of shrinkage estimators can be given and from these a large number of estimators can be generated for different choices of $p$.

\section{MSEs of Different Estimators of Scale Parameter $\theta$}

To obtain the mean squared errors of various estimators of $\theta$ such as $T_{p_{i}},(i=1,2,3,4,5) ; T_{p_{j}}^{*}$, $(j=2,3,4)$ and $T_{k},(k=5,6,7,8,9,10,11,12)$; we write

$$
T=\theta_{0}+W_{(n, p)}\left(\alpha_{(n, p)} \bar{x}-\theta_{0}\right),
$$

where

$$
\begin{aligned}
W_{(n, p)}= & W_{p(i)}, \quad(i=1,2,3,4,5) \\
= & \left(\frac{n-1}{n}\right),\left(\frac{n-2}{n}\right),\left(\frac{n-2}{n+1}\right),\left(\frac{n}{n+1}\right),\left(\frac{n^{2}+n-12}{n^{2}}\right), \frac{(n-1)(n-2)}{n^{2}}, \\
& \frac{(n-1)(n-2)}{n(n+1)},\left(\frac{n-2}{n+1}\right)^{2}
\end{aligned}
$$

and

$$
\alpha_{(n, p)}=1,\left(\frac{n}{n+1}\right)
$$

The MSE of $T$ is given by

$$
\begin{aligned}
& \operatorname{MSE}(T) \\
& =\theta^{2}\left[\frac{W_{(n, p)}^{2}}{n}\left\{\alpha_{(n, p)}^{2}+n\left(\alpha_{(n, p)}-1\right)^{2}\right\}+\left(1-W_{(n, p)}\right)^{2} r^{2}+2 W_{(n, p)}\left(1-W_{(n, p)}\right)\left(\alpha_{(n, p)}-1\right) r\right]
\end{aligned}
$$

Thus the MSEs of the estimators $T_{p_{i}},(i=1,2,3,4,5), T_{p_{j}}^{*},(j=2,3,4)$ and $T_{k},(k=5,6,7,8,9,10$, $11,12)$, can be obtained from $(3.2)$ just by putting the suitable values of $W_{(n, p)}$ and $\alpha_{(n, p)}$.

The optimum value of $r$ that minimizes the MSE of $T$ is given by

$$
r_{o p t}=\frac{\left(\alpha_{(n, p)}-1\right) W_{(n, p)}}{1-W_{(n, p)}}
$$


Table 3.1. MSE's of various estimator of $\theta$

\begin{tabular}{cl}
\hline Estimator & $\left(\frac{\theta^{2}}{(n+1)^{2}}\right)\left\{9 r^{2}-6 r\left(\frac{n-2}{n+1}\right)+\left(\frac{n-2}{n+1}\right)^{2}\right\}$ \\
\hline$T_{10}$ & $\left(\frac{\theta^{2}}{n^{4}}\right)\left\{(3 n-2)^{2} r^{2}-\frac{2 r(n-1)(n-2)(3 n-2)}{n+1}+\frac{(n-1)^{2}(n-2)^{2}}{n+1}\right\}$ \\
\hline$T_{11}$ & $\left(\frac{\theta^{2}}{n^{2}(n+1)^{2}}\right)\left\{\frac{(n-1)^{2}(n-2)^{2}}{n+1}-4(2 n-1)^{2} r^{2}-\frac{4 r(n-1)(n-2)(2 n-1)}{n+1}\right\}$ \\
\hline$T_{12}$ & $\left(\frac{\theta^{2}}{(n+1)^{4}}\right)\left\{9(2 n-1)^{2} r^{2}-\frac{6 r(n-2)^{2}(2 n-1)}{n+1}+\frac{(n-2)^{4}}{n+1}\right\}$ \\
\hline
\end{tabular}

Table 3.2. Optimum values of $r$ and minimum MSE's of various estimators of $\theta$

\begin{tabular}{ccc}
\hline Estimator & Optimum value of $r$ & Minimum MSEs \\
\hline$T_{9}$ & $\frac{n-2}{3(n+1)}$ & $\theta^{2} \frac{n(n-2)^{2}}{(n+1)^{4}}$ \\
\hline$T_{10}$ & $\frac{(n-1)(n-2)}{(3 n-2)(n+1)}$ & $\theta^{2} \frac{(n-1)^{2}(n-2)^{2}}{n^{3}(n+1)^{2}}$ \\
\hline$T_{11}$ & $\frac{(n-1)(n-2)}{2(2 n-1)(n+1)}$ & $\theta^{2} \frac{(n-1)^{2}(n-2)^{2}}{n(n+1)^{4}}$ \\
\hline$T_{12}$ & $\frac{(n-2)^{2}}{3(2 n-1)(n+1)}$ & $\theta^{2} \frac{n(n-2)^{4}}{(n+1)^{6}}$ \\
\hline
\end{tabular}

and hence the minimum MSE of $T$ is given by

$$
\min \operatorname{MSE}(T)=\left(\frac{\theta^{2}}{n}\right) W_{(n, p)}^{2} \alpha_{(n, p)}^{2} .
$$

The optimum value of $r_{\text {opt }}$ can be known as it is function of known quantities $n$ and $p$. We have from (3.2) and (3.4) that

$$
\operatorname{MSE}(T)-\min \operatorname{MSE}(T)=\theta^{2}\left\lfloor r\left(1-W_{(n, p)}\right)+\left(\alpha_{(n, p)}-1\right) W_{(n, p)}\right\rfloor^{2}>0 .
$$

It follows that

$$
\min \operatorname{MSE}(T)<\operatorname{MSE}(T) .
$$

MSE's of different estimators of $\theta$ are provided in Table 3.1.

The 'optimum' values of $r$ (for which the MSE's of $T_{j}$ are minimum) along with the minimum MSE's of $T_{j},(j=9,10,11,12)$ are provided in Table 3.2.

It can easily be shown that the minimum MSE's of these estimators are always fewer than the MMSE estimator $T_{1}=(n /(n+1)) \bar{x}$. It is further, noted that the optimum value of $r$ can be easily known as it is a function of sample size $n$ only.

Conditions under which the estimators $T_{9}, T_{10}, T_{11}$ and $T_{12}$ have smaller mean square errors than that of MMSE estimator $T_{1}$ are presented in Table 3.3 .

Now to have some tangible idea about the performances of these estimators we have computed the relative efficiencies with respect to MMSE estimator $T_{1}$ for different values of $r$ and $n$ and displayed in Table 3.4(a) to Table 3.4(g). Table 3.5 presents the optimum values of $r$ and relative efficiencies of the various estimators of $\theta$. 
Table 3.3. Conditions under which various estimators have smaller MSE's than that of MMSE estimator $T_{1}$

\begin{tabular}{cl}
\hline Estimator & Condition \\
\hline$T_{9}$ & $\left\{3 r^{2}-2 r\left(\frac{n-2}{n+1}\right)-\left(\frac{2 n-1}{n+1}\right)\right\} \leq 0$ \\
\hline$T_{10}$ & $\left\{r^{2}-\frac{2(n-1)(n-2) r}{(3 n-2)(n+1)}-\frac{6 n^{3}-13 n^{2}+12 n-4}{(n+1)(3 n-2)^{2}} \leq 0\right.$ \\
\hline$T_{11}$ & $\left\{r^{2}-\frac{(n-1)(n-2) r}{(2 n-1)(n+1)}-\frac{2 n^{3}-3 n^{2}+3 n-1}{(n+1)(2 n-1)^{2}} \leq 0\right.$ \\
\hline$T_{12}$ & $\left\{r^{2}-\frac{3(n-2)^{2} r}{(2 n-1)(n+1)}-\frac{12 n^{3}-18 n^{2}+28 n-15}{9(n+1)(2 n-1)^{2}} \leq 0\right.$ \\
\hline
\end{tabular}

It is observed from Table 3.4(a) to Table $3.4(\mathrm{~g})$ that when $|r| \leq 0.3$, the estimator $T_{12}$ is better than the estimators $\left(T_{3}, T_{4}\right),\left(T_{5}, T_{6}, T_{7}\right)$ and $T_{8}$ suggested by Pandey (1983), Pandey and Srivastava (1985) and Jani (1991) respectively for all $n$ except for $(r=-0.3, n=3)$.

Further, it is noticed that the estimators $T_{12}$ seem to be the most promising among all the estimators for $|r| \leq 0.1$ and all values of $n$. However, the performances of the estimators $T_{9}, T_{10}$ and $T_{11}$ are also appreciable in respect to $T_{j},(j=3$ to 8$)$ when $r$ range, between -0.3 to 0.3 and $n \leq 9$.

Table 3.5 demonstrates that the estimator $T_{12}$ is better than the rest of the estimators when $r$ attains its optimum value.

This study reveals that there is enough scope to choose simple estimators better than those considered by Pandey (1983), Pandey and Srivastava (1985) and Jani (1991) and MMSE estimator $T_{1}$.

REMARK 3.1. Similar classes of estimators can be further defined for the data based on the failure censored sample (Jani, 1991, Section 6, p.70).

REMARK 3.2. Similarly, alternative classes of estimators to the class of estimators

$$
\hat{\theta}_{(p)}=\theta_{0}+\alpha_{(p)}\left(\hat{\theta}-\theta_{0}\right)
$$

for the scale parameter $\theta$ and hence alternative to the class of estimators

$$
\hat{\mu}_{(p)}=x_{(1)}+\left(\frac{n-1}{n}\right) \hat{\theta}_{(p)}
$$

of the mean $\mu$ of a 2-parameter exponential distribution

$$
s f\{x ; \theta, \lambda\}=\exp \left\{-\frac{x-\lambda}{\theta}\right\}, \quad x \geq \lambda
$$

can be derived and studied their properties, where $\lambda, \theta$ (location, scale) parameter, $\theta>0$,

$$
\hat{\theta}=\frac{1}{n-1} \sum_{i=1}^{n}\left(x_{i}-x_{(1)}\right) .
$$

$x_{(1)}: \min \left(x_{i}, i=1,2, \ldots, n\right):$ minimum order statistic,

$$
\alpha_{(p)}=\frac{\sqrt{n-1-p}}{(n-1)^{p} \sqrt{n-1-2 p}}
$$

where $p$ is a real number such that $p \in(-\infty,(n-1) / 2)$. The class of estimators $\hat{\theta}_{(p)}$ and $\hat{\mu}_{(p)}$ are due to Kourouklis (1994). 
Table 3.4. Relative efficiencies of various estimators of $\theta$ with respect to MMSE estimator $T_{1}=(n /(n+1)) \bar{x}$ at different values of $r$

\begin{tabular}{|c|c|c|c|c|c|c|c|c|c|c|c|}
\hline \multicolumn{12}{|c|}{ (a) $r=-0.5$} \\
\hline \multirow{2}{*}{$\begin{array}{c}\text { Sample } \\
\text { size } n\end{array}$} & \multicolumn{11}{|c|}{ Estimator } \\
\hline & $T_{1}$ & $T_{3}$ & $T_{4}$ & $T_{5}$ & $T_{6}$ & $T_{7}$ & $T_{8}$ & $T_{9}$ & $T_{10}$ & $T_{11}$ & $T_{12}$ \\
\hline 3 & 1.00 & 1.22 & 1.14 & 1.69 & 1.42 & - & 1.23 & 1.23 & 1.21 & 1.16 & 1.06 \\
\hline 5 & 1.00 & 1.10 & 1.07 & 1.49 & 1.20 & 1.35 & 1.14 & 1.14 & 1.13 & 1.06 & 0.91 \\
\hline 7 & 1.00 & 1.05 & 1.05 & 1.34 & 1.13 & 1.06 & 1.10 & 1.10 & 1.10 & 1.03 & 0.88 \\
\hline 9 & 1.00 & 1.01 & 1.00 & 1.26 & 1.10 & 0.96 & 1.08 & 1.08 & 1.08 & 1.02 & 0.87 \\
\hline 15 & 1.00 & 0.89 & 0.89 & 1.15 & 1.06 & 0.91 & 1.05 & 1.05 & 1.05 & 1.01 & 0.87 \\
\hline \multicolumn{12}{|c|}{ (b) $r=-0.3$} \\
\hline Sample & \multicolumn{11}{|c|}{ Estimator } \\
\hline size $n$ & $T_{1}$ & $T_{3}$ & $T_{4}$ & $T_{5}$ & $T_{6}$ & $T_{7}$ & $T_{8}$ & $T_{9}$ & $T_{10}$ & $T_{11}$ & $T_{12}$ \\
\hline 3 & 1.00 & 1.69 & 1.50 & 3.25 & 1.58 & - & 1.29 & 1.36 & 2.70 & 2.77 & 2.81 \\
\hline 5 & 1.00 & 1.52 & 1.43 & 1.93 & 1.26 & 1.51 & 1.18 & 1.87 & 1.90 & 2.01 & 2.09 \\
\hline 7 & 1.00 & 1.38 & 1.32 & 1.56 & 1.17 & 1.08 & 1.13 & 1.58 & 1.59 & 1.69 & 1.78 \\
\hline 9 & 1.00 & 1.37 & 1.35 & 1.40 & 1.12 & 0.97 & 1.10 & 1.44 & 1.44 & 1.52 & 1.60 \\
\hline 15 & 1.00 & 1.07 & 1.06 & 1.21 & 1.07 & 0.91 & 1.06 & 1.25 & 1.25 & 1.30 & 1.36 \\
\hline \multicolumn{12}{|c|}{ (c) $r=-0.1$} \\
\hline \multirow{2}{*}{$\begin{array}{c}\text { Sample } \\
\text { size } n\end{array}$} & \multicolumn{11}{|c|}{ Estimator } \\
\hline & $\overline{T_{1}}$ & $\overline{T_{3}}$ & $\overline{T_{4}}$ & $\overline{T_{5}}$ & $\overline{T_{6}}$ & $T_{7}$ & $T_{8}$ & $T_{9}$ & $T_{10}$ & $T_{11}$ & $T_{12}$ \\
\hline 3 & 1.00 & 1.97 & 1.70 & 6.03 & 1.67 & - & 1.32 & 8.16 & 9.25 & 12.00 & 19.69 \\
\hline 5 & 1.00 & 1.97 & 1.80 & 2.27 & 1.29 & 1.60 & 1.20 & 3.18 & 3.37 & 4.36 & 7.48 \\
\hline 7 & 1.00 & 1.97 & 1.85 & 1.70 & 1.18 & 1.08 & 1.14 & 2.23 & 2.30 & 2.83 & 4.35 \\
\hline 9 & 1.00 & 1.94 & 1.84 & 1.48 & 1.14 & 0.97 & 1.11 & 1.85 & 1.89 & 2.23 & 3.16 \\
\hline 15 & 1.00 & 1.87 & 1.78 & 1.24 & 1.08 & 0.91 & 1.07 & 1.44 & 1.45 & 1.61 & 2.00 \\
\hline \multicolumn{12}{|c|}{ (d) $r=0.0$} \\
\hline \multirow{2}{*}{$\begin{array}{c}\text { Sample } \\
\text { size } n\end{array}$} & \multicolumn{11}{|c|}{ Estimator } \\
\hline & $T_{1}$ & $T_{3}$ & $T_{4}$ & $T_{5}$ & $T_{6}$ & $T_{7}$ & $T_{8}$ & $T_{9}$ & $T_{10}$ & $T_{11}$ & $T_{12}$ \\
\hline 3 & 1.00 & 1.98 & 1.71 & 6.03 & 1.68 & - & 1.32 & 16.00 & 20.25 & 36.00 & 25.00 \\
\hline 5 & 1.00 & 1.99 & 1.84 & 2.32 & 1.30 & 1.61 & 1.13 & 4.00 & 4.30 & 6.25 & 16.00 \\
\hline 7 & 1.00 & 2.05 & 1.82 & 1.72 & 1.19 & 1.09 & 1.12 & 2.56 & 2.67 & 3.49 & 6.55 \\
\hline 9 & 1.00 & 2.04 & 1.79 & 1.48 & 1.14 & 0.97 & 1.11 & 2.04 & 2.09 & 2.58 & 4.17 \\
\hline 15 & 1.00 & 2.03 & 1.71 & 1.25 & 1.08 & 0.91 & 1.07 & 1.52 & 1.53 & 1.74 & 2.30 \\
\hline \multicolumn{12}{|l|}{ (e) $r=0.1$} \\
\hline \multirow{2}{*}{$\begin{array}{c}\text { Sample } \\
\text { size } n\end{array}$} & \multicolumn{11}{|c|}{ Estimator } \\
\hline & $T_{1}$ & $T_{3}$ & $T_{4}$ & $T_{5}$ & $T_{6}$ & $T_{7}$ & $T_{8}$ & $T_{9}$ & $T_{10}$ & $T_{11}$ & $T_{12}$ \\
\hline 3 & 1.00 & 1.88 & 1.63 & 6.03 & 1.67 & - & 1.32 & 21.05 & 25.63 & 36.00 & 36.57 \\
\hline 5 & 1.00 & 1.89 & 1.74 & 2.27 & 1.29 & 1.60 & 1.20 & 4.65 & 5.09 & 7.49 & 17.02 \\
\hline 7 & 1.00 & 1.93 & 1.80 & 1.70 & 1.18 & 1.08 & 1.14 & 2.82 & 2.95 & 3.93 & 7.43 \\
\hline 9 & 1.00 & 1.85 & 1.79 & 1.48 & 1.14 & 0.97 & 1.11 & 2.19 & 2.25 & 2.82 & 4.63 \\
\hline 15 & 1.00 & 1.75 & 1.71 & 1.24 & 1.08 & 0.91 & 1.07 & 1.57 & 1.59 & 1.83 & 2.44 \\
\hline \multicolumn{12}{|c|}{ (f) $r=0.3$} \\
\hline Sample & & & & & & Estim & & & & & \\
\hline size $n$ & $T_{1}$ & $T_{3}$ & $T_{4}$ & $T_{5}$ & $T_{6}$ & $T_{7}$ & $T_{8}$ & $T_{9}$ & $T_{10}$ & $T_{11}$ & $T_{12}$ \\
\hline 3 & 1.00 & 1.45 & 1.32 & 3.25 & 1.58 & - & 1.29 & 6.56 & 6.12 & 5.14 & 3.51 \\
\hline 5 & 1.00 & 1.35 & 1.29 & 1.93 & 1.26 & 1.51 & 1.18 & 4.26 & 2.59 & 4.75 & 3.94 \\
\hline 7 & 1.00 & 1.31 & 1.27 & 1.56 & 1.17 & 1.08 & 1.13 & 2.85 & 2.93 & 3.41 & 3.61 \\
\hline 9 & 1.00 & 1.31 & 1.29 & 1.40 & 1.12 & 0.97 & 1.10 & 2.25 & 2.30 & 2.67 & 3.08 \\
\hline 15 & 1.00 & 1.13 & 1.07 & 1.21 & 1.07 & 0.91 & 1.06 & 1.62 & 1.63 & 1.82 & 2.14 \\
\hline (g) $r=0$. & & & & & & & & & & & \\
\hline Sample & & & & & & Estim & & & & & \\
\hline size $n$ & $T_{1}$ & $T_{3}$ & $T_{4}$ & $T_{5}$ & $T_{6}$ & $T_{7}$ & $T_{8}$ & $T_{9}$ & $T_{10}$ & $T_{11}$ & $T_{12}$ \\
\hline 3 & 1.00 & 1.10 & 1.07 & 1.69 & 1.42 & - & 1.23 & 2.29 & 2.08 & 1.71 & 1.21 \\
\hline 5 & 1.00 & 1.03 & 1.02 & 1.49 & 1.20 & 1.35 & 1.14 & 2.67 & 2.59 & 2.17 & 3.94 \\
\hline 7 & 1.00 & 0.93 & 0.93 & 1.34 & 1.13 & 1.06 & 1.10 & 2.29 & 2.28 & 2.13 & 1.52 \\
\hline 9 & 1.00 & 0.80 & 0.86 & 1.26 & 1.10 & 0.96 & 1.08 & 1.98 & 1.98 & 1.97 & 1.56 \\
\hline 15 & 1.00 & 0.80 & 0.80 & 1.15 & 1.06 & 0.91 & 1.05 & 1.54 & 1.55 & 1.60 & 1.49 \\
\hline
\end{tabular}


Table 3.5. Optimum values of $r\left\{=\left(\theta_{0} / \theta-1\right)\right\}$ and relative efficiencies of various estimator of $\theta$ with respect to MMSE estimator $T_{1}=(n /(n+1)) \bar{x}$ for various values of $n$

\begin{tabular}{|c|c|c|c|c|c|c|}
\hline \multirow{2}{*}{\multicolumn{2}{|c|}{ Estimator }} & \multicolumn{5}{|c|}{ Sample size $n$} \\
\hline & & 3 & 5 & 7 & 9 & 15 \\
\hline \multirow{2}{*}{$T_{9}$} & $t_{\text {opt }}$ & 0.083 & 0.1667 & 0.2083 & 0.2333 & 0.2708 \\
\hline & $R E$ & 21.33 & 4.80 & 2.93 & 2.27 & 1.62 \\
\hline \multirow{2}{*}{$T_{10}$} & $t_{o p t}$ & 0.07143 & 0.10256 & 0.14423 & 0.16471 & 0.26454 \\
\hline & $R E$ & 27.00 & 5.10 & 3.05 & 2.33 & 1.63 \\
\hline \multirow{2}{*}{$T_{11}$} & $t_{\text {opt }}$ & 0.0500 & 0.1111 & 0.14423 & 0.16471 & 0.19612 \\
\hline & $R E$ & 48.00 & 7.50 & 3.98 & 2.87 & 1.86 \\
\hline \multirow{2}{*}{$T_{12}$} & $t_{o p t}$ & 0.01667 & 0.05556 & 0.08013 & 0.09608 & 0.12141 \\
\hline & $R E$ & 341.39 & 19.20 & 7.49 & 4.63 & 2.45 \\
\hline
\end{tabular}

\section{Acknowledgement}

Authors are thankful to the referees for their valuable suggestions in regards to the improvement of the paper.

\section{References}

Jani, P. N. (1991). A class of shrinkage estimators for the scale parameter of the exponential distribution, IEEE Transactions on Reliability, 40, 68-70.

Kourouklis, S. (1994). Estimation in the 2-parameter exponential distribution with prior information, IEEE Transactions on Reliability, 43, 446-450.

Pandey, B. N. (1983). Shrinkage estimation of the exponential scale parameter, IEEE Transactions on Reliability, 32, 203-205.

Pandey, B. N. and Srivastava, R. (1985). On shrinkage estimation of the exponential scale parameter, IEEE Transactions on Reliability, 34, 224-226.

Thompson, J. R. (1968). Some shrinkage technique for estimating the mean, Journal of the American Statistical Association, 63, 113-123. 